\title{
Multi-excitation fluorescence spectroscopy for analysis of non-alcoholic fatty liver disease
}

\author{
Vincent R. Sauvage, MSc ${ }^{1,2}$, Adam P. Levene, $\mathrm{MBChB}^{3}$, Hoa T. Nguyen, $\mathrm{MSc}^{2,4}$, Tobias C. Wood \\ MEng ${ }^{1,5}$, Hiromi.Kudo, MSc ${ }^{3}$, Danilo Concas, $\mathrm{PhD}^{6}$, Howard C. Thomas, $\mathrm{PhD}^{7}$, Mark R. Thursz, \\ $\mathrm{PhD}^{7}$, Robert D. Goldin, MD ${ }^{3}$, Quentin M. Anstee, $\mathrm{PhD}^{6,8}$ and Daniel S. Elson, $\mathrm{PhD}^{1,2}$ \\ ${ }^{1}$ Department of Surgery and Cancer, Imperial College London, London SW7 2AZ, UK. (VRS, DSE) \\ ${ }^{2}$ Institute of Biomedical Engineering, Imperial College London, London SW7 2AZ, UK. (VRS, HTN, DSE) \\ ${ }^{3}$ Department of Histopathology, Imperial College London, London SW7 2AZ, UK. (APL, HK, RDG) \\ ${ }^{4}$ Institut d'Alembert, École Normale Supérieure de Cachan, Cachan 94235, France. (HTN) \\ ${ }^{5}$ Department of Computing, Imperial College London, SW7 2AZ, UK. (TCW) \\ ${ }^{6}$ Liver Research Group, MRC Mammalian Genetics Unit, MRC Harwell, Oxfordshire OX11 ORD, UK. (DC, QMA) \\ ${ }^{7}$ Department of Gastroenterology \& Hepatology, Imperial College London, London SW7 2AZ, UK. (HCT, MRT) \\ ${ }^{8}$ Liver Research Group, Institute of Cellular Medicine, Newcastle University, Newcastle-Upon-Tyne NE1 7RU, UK. (QMA) \\ Contract grant sponsor: Elements of this work have been funded by the EPSRC grants DT/F003064/1 and EP/E06342X/1 \\ to DSE and an MRC Programme Grant to QMA, RDG \& MT. QMA is a Newcastle NIHR BRC Investigator, RG and MRT \\ are Imperial College NIHR BRC Investigators. \\ Correspondence to: Daniel Elson, Department of Surgery and Cancer, Imperial College London, South Kensington \\ Campus, SW7 2AZ, London, United Kingdom. Email: ds.elson@imperial.ac.uk
}

\section{Abstract}

Background and objectives: the increasing incidence of non-alcoholic fatty liver diseases (NAFLD) and the consequent progression to cirrhosis is expected to become a major cause of liver transplantation. This will exacerbate the organ donor shortage and mean that 'marginal' fatty liver grafts are more frequently used. Autofluorescence spectroscopy is a fast, objective and nondestructive method to detect change in the endogenous fluorophores distribution and could prove to be a valuable tool for NAFLD diagnosis and transplant graft assessment.

Materials and methods: A system was constructed consisting of a fibre probe with two laser diodes that provided excitation light at $375 \mathrm{~nm}$ and $405 \mathrm{~nm}$, and an imaging spectrograph system. This was used to distinguish fluorescence spectra acquired from the harvested livers from mice with NAFLD of differing severity (healthy, mild steatosis and steatohepatitic). The fluorescence data was entered into a sparse multiclass probabilistic algorithm for disease classification. Histopatholagy, TBARS and ALT assays were conducted in addition to the fluorescence measurements

Results: Thiobarbituric Acid reactive substances (TBARS) and Alanine transaminase (ALT) assays enabled differentiation of the steatohepatitic group from the mild steatosis and control groups ( $p \leq 0.028$ ) but failed to separate the mild steatotic group from the control group. The three groups were all clearly differentiated from each other using fluorescence spectroscopy, and classification accuracy was found to be $95 \%$.

Conclusion: Fluorescence spectroscopy appears to be a promising approach for the analysis of diseased liver tissue. 


\section{Introduction}

Non-Alcoholic Fatty Liver Disease (NAFLD) represents a spectrum of progressive liver disease occurring in the absence of significant alcohol consumption, ranging from steatosis (fatty liver), through steatohepatitis (fat plus inflammation) to liver fibrosis, and ultimately cirrhosis [1,2]. NAFLD is associated with obesity and insulin resistance/diabetes and is characterized by an accumulation of triglycerides (fat) in the liver. Although steatosis has traditionally been considered a relatively benign condition [3], its evolution into more serious progressive fibrosis and cirrhosis of the liver is a concern. NAFLD has become the third most important indication for liver transplantation and is expected to become the leading indication in future decades [4). Worldwide, the prevalence of NAFLD is estimated to be between 10 and 39\% [5]. Its prevalence reaches $57 \%$ to $74 \%$ among the obese population, $50 \%$ among diabetic patients [6] and the overall prevalence is set to rise with the worldwide epidemic of obesity and diabetes (the obese adult population will rise from at least 400 million in 2005 to 700 million by 2013 [7], while the diabetes population is expected to grow from 285 million in 2010 to 438 million in 2030 [8] according to the $\mathrm{WHO}$ ).

Given the shortage of organ donors, and the high prevalence of NAFLD within the population, the use of steatotic (fatty) 'marginal' grafts is increasingly common in liver transplantation [9]. However, severe steatosis and steatohepatitis [10,11] are associated with increased risk of primary graft non-function. Consequently, to aid effective organ selection, there is a need to rapidly and robustly assess donor organs for degree of steatosis and the presence of significant oxidative stress, as these may indicate grafts at greater risk of non-function after transplantation.

At present there is no rapid and accurate test to diagnose NAFLD or non-alcoholic steatohepatitis (NASH). For example, blood biochemistry is insensitive and non-specific, whilst biopsy is timeconsuming and not well suited to peri/intraoperative assessment of fatty liver [12]. Several noninvasive imaging techniques have been tested for NAFLD diagnosis. Ultrasound detects steatosis with a limited sensitivity $(67 \%)$ and specificity $(77 \%)$ [13], but gives better results when the severity of the steatosis increases [14]. CT imaging enables a sensitivity of $100 \%$ and a specificity of $82-93 \%$ (10) for steatosis diagnosis. MRI allows the most accurate fat quantification and hence enables the best sensitivity (100\%) and specificity (92\%) for steatosis diagnosis [15]. However, because all of these techniques measure the triglycerides, they can only assess the severity of steatosis (fat content) and are unable to differentiate steatosis from steatohepatitis or cirrhosis since they are characterized by tissue inflammation and fibrosis rather than by triglyceride concentration. In fact, when steatohepatitis and cirrhosis are well established the liver, fat content may decrease, potentially leading to an underestimation of the disease severity [16]. Therefore, a fast and minimally invasive method able to differentiate normal tissue from steatosis and from steatohepatitis would be desirable.

Fluorescence spectroscopy has been extensively used for cancer diagnosis in various organs because it provides a fast, objective, minimally invasive (as the instrumentation can be 
miniaturised and does not use harmful or ionising radiation) and less traumatic alternative to histopathology [17]. Fluorescence spectroscopy has several potential applications in liver tissue characterisation. In an ex vivo setting it could be an alternative or a complement to histopathology to assess liver biopsies and would also enable non-destructive detection of NAFLD in liver transplant grafts which are more prone to primary non-function. In vivo, it could replace biopsy in patients for whom biopsy would be a high risk procedure. It could also be used as an adjunct in patients already undergoing a surgical procedure (bariatric surgery) or to assess the donor organ at harvest.

Autofluorescence from liver has previously been studied by Croce et al [18-21] in a laboratory microscopy setting. In this work, it was shown that $\mathrm{NAD}(\mathrm{P}) \mathrm{H}$, vitamin $\mathrm{A}$ and flavins were major contributors to liver autofluorescence when excited at $366 \mathrm{~nm}$. In particular, it was shown that accumulation of lipids led to an increased presence of the fluorophore, vitamin A. Also, steatohepatitis can be accompanied by fibrosis, a proliferation of the connective tissue whose main component is fluorescent collagen. Other contributors to the liver autofluorescence are likely to be flavins, riboflavin, arachinoid acid, lipopigments, and porphyrins [22-25], all found to be present in liver and emitting in the visible range [26]. Thus, the construction of a fluorescence spectroscopy probe system seems a promising candidate to non-invasively diagnose and characterise steatosis and steatohepatitis.

In this study a fluorescence spectroscopy probe was constructed that could collect fluorescence emission spectra at two excitation wavelengths. This could excite different subspecies of the endogenous fluorophores present and the emission spectra at different excitation wavelengths could then be used to distinguish different tissue states. A sparse multinomial logistic regression enabled differences between normal, steatotic and steatohepatitic livers to be detected in an animal model.

\section{Material and methods}

\section{Multi-excitation fluorescence spectroscopy system}

The violet laser fluorescence system combined two laser diodes emitting at $375 \mathrm{~nm}$ and $405 \mathrm{~nm}$ and a custom made probe (Romack, Inc) consisting of six hexagonally packed fluorescence collection fibres surrounding an excitation fibre that delivered the laser light to the sample (figure 1). The diameter and numerical aperture of the fibres were $200 \mu \mathrm{m}$ and 0.22 respectively and they were chosen to have low autofluorescence and attenuation in the near ultraviolet spectral region. The probe distal tip was covered by a glass window and the fibres and window were housed in a stainless steel tube with an external diameter of $2 \mathrm{~mm}$. This could enable it to be inserted into endoscope working channels, needles or small orifices. The proximal end was divided into two arms, the first one contained the excitation fibre and was coupled to the laser optics using an SMA connector; the second arm included the six emission fibres arranged in a linear array inside another SMA connector. 
The laser light from the two laser diodes was collimated by $4.6 \mathrm{~mm}$ focal length lenses and the beams were combined using a dichroic mirror so that both beams could be coupled into the excitation fibre using an $8 \mathrm{~mm}$ achromatic lens, with two steering mirrors used to align one of the beams and ensure coaxial propagation. Two beam shutters (one for each laser diode) enabled the selection of the excitation wavelength delivered to the sample. The optical power at the distal end of the probe was $3.5 \mathrm{~mW}$ for both lasers.

The fluorescence emission from the six fibres was focussed onto the $80 \mu \mathrm{m}$ wide input slit of an imaging spectrograph (Specim, Ltd) using an achromatic lens doublet (60 mm and $30 \mathrm{~mm}$ ). A 430 $\mathrm{nm}$ long-pass filter was inserted between these two lenses to block the light of the laser diodes reflected by the sample. The light dispersed by the prism-grating-prism element of the spectrograph was then acquired with a sensitive cooled CCD camera (Retiga EXI, Qimaging, $1392 \times 1040$ pixels). All the optics were assembled together using a cage system and a breadboard so that the system was robust and portable. A LabVIEW program controlled the exposure time $(100 \mathrm{~ms})$ and beam shutters and enabled acquisition of fluorescent signals from the laser diodes sequentially with an adjustable number of measurements.

\section{Study design and animal husbandry}

Research was approved by the local ethics committee and performed in accordance with the UK Animal (Scientific Procedures) Act 1986. Mice were housed under standard conditions and provided with a commercial chow (\#801722, SDS, UK) and free access to water. The study was conducted to investigate the autofluorescence spectroscopy of liver tissue from mice with varying degrees of NAFLD and used mouse models with steatosis and steatohepatitis induced by a combination of genetic and dietary modulation [1].

8-week old male non-obese heterozygote $\mathrm{Db} / \mathrm{m}$ control mice were fed with a control diet (BKSCg-m +/- Lepr ${ }^{\mathrm{db}} / \mathrm{J}$, Charles River, Belgium - 'control group'). This was compared to genetically obese $\mathrm{Db} / \mathrm{Db}$ mice (BKS-Cg-m +/+ Lepr ${ }^{\mathrm{db}} / \mathrm{J}$, Charles River, Belgium) fed either with a nutritionally replete control diet (\#A02082003B - 'mild steatosis group') or with a choline deficient diet (\#A02082002B, Research Diets, USA - 'steatohepatitis group'). There were initially five animals per group although during the study two of the mice from the mild steatosis group became unwell and had to be culled early and were excluded from the study.

At the end of the study, animals were culled by exsanguination under general anaesthesia and liver tissue was quickly harvested. The liver was bisected and half was immediately snap-frozen in liquid nitrogen for subsequent biochemical analysis. Blood was also obtained for biochemical analysis. Without any treatment or staining, liver tissues were submitted to measurements immediately after culling to minimize air-exposure effects, cleaned of blood to minimize haemoglobin absorption and brought from the operating table to the optical bench. Emission spectra were recorded and the livers were then fixed in formalin solution for further histopathological study. 
Data was acquired at between three and five different sites on each liver. At each site six measurements were recorded from the six emission fibres in the probe, probing an overlapping but slightly different tissue volume. This leads to a total of 114 measurements for the normal group, 72 measurements from the steatotic group, and 114 measurements for the steatohepatitic group (note that one measurement consists of two emission spectra - one for $375 \mathrm{~nm}$ and one for $405 \mathrm{~nm}$ excitation).

\section{Data processing and analysis}

The six emission spectra recorded for each excitation wavelength were extracted from the CCD images and the intensities at different spatial locations on the image were converted into spectra using calibration data obtained with a number of specific laser wavelengths. The spectrum of a tungsten-halogen calibrated light source (Ocean Optics Inc.) was also recorded at the start of the experiment to take into account the system spectral response and the ratio of this spectrum over the real spectrum was used to correct the raw spectra. Spectra were normalized by the area under the curve and truncated between 450 and $750 \mathrm{~nm}$. The spectra from the two excitation wavelengths were concatenated prior to processing for each measurement.

Sparse multinomial logistic regression (SMLR), as previously developed by Krishnapuram et al. [27], was used to separate the spectral features into three classes: control, mild steatosis and steatohepatitis with an open source software package [28]. SMLR belongs to the family of supervised learning algorithms; it proposes a true multiclass classification of the spectra using classifiers built as a linear combination of the input features, i.e the 106 fluorescence intensities at the 106 wavelengths sampled between $450 \mathrm{~nm}$ and $750 \mathrm{~nm}$ comprising each spectrum. SMLR has previously been demonstrated to be highly effective in differentiating autofluorescence and diffuse reflectance spectra from healthy, marginal, and tumourous brain tissue $[29,30]$. The SMLR classification was performed here with a K-fold cross validation rather than with a leave-one-out cross validation since the six spectra from one acquisition cannot be considered to be totally independent. In this approach, the sample pool of 300 spectra was divided into 50 subsets $(K=50)$ each containing the six spectra from the same acquisition so that during the classifier learning groups of six were held out at a time.

The probability that a spectrum belonged to a certain class is assumed to follow the multinomial logistic regression model where the regression coefficients are the classifier weight vectors of the corresponding class. The spectrum was assumed to belong to the class whose weight vector maximised this probability. The estimation of the weight matrix (106 lines, three columns) for the three different classes (in other words the result of the classifier learning process), was calculated from a maximum a posteriori estimate, with the spectra as input training data. This approach contains a number of advantages over traditional maximum likelihood estimation, as described in $[27,31]$. 


\section{Histopathology}

Formalin fixed liver tissue was processed into paraffin wax and sections were stained with H\&E. H\&E stained sections were assessed using the validated NASH Clinical Research Network histological classification system. Steatosis was scored 0-3 where score $0=<5 \%$ steatosis, $1=5$ $33 \%$ steatosis, $2=33-66 \%$ steatosis, and $3=>66 \%$ steatosis. Lobular inflammation was scored $0-3$ where score $0=$ none, $1=<2$ foci per $\times 200$ field, $2=2-4$ foci per $\times 200$ field, and $3=>4$ foci per x200 field. Finally hepatocyte ballooning was scored $0-2$ where score $0=$ none, $1=$ few ballooned cells, and $2=$ prominent ballooning. These scores were then added together to give a NAFLD activity score (NAS) which decides whether a histological diagnosis of NASH is made ( $\geq 5$ is definite NASH) [32].

\section{TBARS and ALT biochemistry}

The MCD mouse model of NASH is characterised by increased reactive oxygen species production. To quantify this, a TBARS assay was performed. Free radical damage ('tissue oxidative stress') is a major driver of disease pathogenesis, inflammation and progression in NASH. TBARS is not used in standard practice but is an accepted research tool to determine lipid peroxidation as a surrogate measure for free radical damage within the liver. Inclusion in this study is to provide a comparator directly related to disease pathogenesis beyond that which would be available in routine practice. In practice, the clinical diagnosis of NASH is based on the identification of raised alanine transaminase levels (indicating inflammation) in patients with features of the metabolic syndrome (e.g. obesity, diabetes and dyslipidaemia) with supporting evidence of fatty liver on ultrasound imaging. Approximately $100 \mathrm{mg}$ of frozen liver was placed into a ceramic beads tube. Then $300 \mu$ l of RIPA buffer was added and the tubes placed into a Precellys rotor at $6500 \mathrm{rpm}$ for 1 cycle of 50 seconds to homogenise the samples. The supernatant was then kept on ice and used in the assay. $100 \mu \mathrm{l}$ of the lysate was placed into a $1.5 \mathrm{ml}$ microcentrifuge tube and $200 \mu \mathrm{l}$ of ice cold $10 \%$ Trichloracetic acid was added. The sample was then incubated for 15 minutes on ice followed by centrifugation at $3000 \mathrm{~g}$ for 10 minutes. Standards were prepared using distilled water and $500 \mu \mathrm{M}$ Malondialdehyde (MDA) to give known MDA concentrations from $0 \mu \mathrm{M}$ to $50 \mu \mathrm{M}$. $300 \mu \mathrm{l}$ of the samples and standards were then mixed with $300 \mu \mathrm{l}$ of $0.67 \%$ thiobarbituric acid and incubated at $95{ }^{\circ} \mathrm{C}$ for 10 minutes. The samples were then allowed to chill.

Using a 96 well plate $150 \mu \mathrm{l}$ of each standard and sample was placed into a well, performed in duplicate. Spectrophotometry at $532 \mathrm{~nm}$ was performed using a plate reader. Using the absorbance values and standard curve the MDA concentration in $\mu \mathrm{M}$ per $100 \mathrm{mg}$ of liver was calculated for each sample.

The blood obtained when the mice were culled was placed in a plasma collection tube which when centrifuged at $3000 \mathrm{rpm}$ for 10 minutes separated the plasma from the erythrocytes. The 
supernatant plasma was removed from the blood and stored at $-80^{\circ} \mathrm{C}$ until analysed in an automated biochemical analyser to give ALT measurements in IU/L.

\section{RESULTS}

\section{Histopathology}

H\&E stained sections showed that the three groups could be clearly distinguished. The nonobese $\mathrm{Db} / \mathrm{m}$ mice fed chow ('control group') had normal liver histology. The $\mathrm{Db} / \mathrm{Db}$ mice fed chow ('mild steatosis group') exhibited features of isolated steatosis without lobular inflammation and the Db/Db mice fed a MCD diet ('steatohepatitis group') had NASH with marked steatosis, moderate lobular inflammation and many ballooned cells (see Table 1 and Figure 2).

\section{TBARS Assay}

There were significantly raised MDA concentrations in the livers of $D b / D b$ mice on MCD diet (steatohepatitis group) compared to the two other study groups, as expected $-p=0.028$ with $\mathrm{Db} / \mathrm{Db}$ mice on standard chow (control group) and $\mathrm{p}=0.011$ with $\mathrm{Db} / \mathrm{m}$ mice on standard chow (mild steatosis group), see Figure 3. The mean MDA concentrations ( $\mu \mathrm{M}$ per $100 \mathrm{mg}$ of liver) were $0.26 \pm 0.02$ for the $\mathrm{Db} / \mathrm{m}$ mice on standard chow, $0.28 \pm 0.02$ for the $\mathrm{Db} / \mathrm{Db}$ mice on standard chow and $1.79 \pm 0.46$ for the $\mathrm{Db} / \mathrm{Db}$ mice on MCD diet (Table 2). These results confirm that there was increased oxidative stress within the liver of the Db/Db mice on the MCD diet. No significant difference was detected between the chow fed $\mathrm{Db} / \mathrm{Db}$ mice with isolated steatosis or the phenotypically normal $\mathrm{Db} / \mathrm{m}$ mice fed chow (Figure 3).

\section{ALT Biochemistry}

There were significantly increased ALT levels in the plasma of the $\mathrm{Db} / \mathrm{Db}$ mice fed MCD diet compared to the two other study groups, as expected $(p=<0.001$ for $D b / D b$ mice on standard chow and $\mathrm{Db} / \mathrm{m}$ mice on standard chow - see Figure 4). The mean ALT levels in the plasma (IU/L) were $26.40 \pm 4.07$ for the $\mathrm{Db} / \mathrm{m}$ mice on standard chow, $91.77 \pm 4.78$ for the $\mathrm{Db} / \mathrm{Db}$ mice on standard chow and $307.34 \pm 33.38$ for the $\mathrm{Db} / \mathrm{Db}$ mice on MCD diet (table 3 ). These results confirm that there was biochemical hepatitis within the liver of the Db/Db mice on the MCD diet which is in keeping with the lobular inflammation and ballooning identified on H\&E stain. No significant difference was detected between the chow fed $\mathrm{Db} / \mathrm{Db}$ mice with isolated steatosis or the phenotypically normal $\mathrm{Db} / \mathrm{m}$ mice fed chow although there does appear to be a trend towards increased ALT in the steatotic Db/Db mice.

\section{Fluorescence spectroscopy results}

Mean spectra at $375 \mathrm{~nm}$ and $405 \mathrm{~nm}$ excitation and error bars representing data standard deviation are presented in Figure 5. At $375 \mathrm{~nm}$ excitation, liver autofluorescence peaked at around $475 \mathrm{~nm}$ which is attributed to the combined fluorescence of $\mathrm{NAD}(\mathrm{P}) \mathrm{H}$ (emission at around 
$475 \mathrm{~nm}$ ) and vitamin A (emission at around $490 \mathrm{~nm}$ ) [20]. Mild steatotic tissue showed relatively higher $475 \mathrm{~nm}$ intensity than healthy tissue, with intensity increasing in conjunction with the severity of the steatosis in these two groups. In steatohepatitis the tissue exhibited lower peak fluorescence than healthy and steatotic tissue. The $405 \mathrm{~nm}$ excitation spectra revealed a peak intensity at $500 \mathrm{~nm}$. A secondary peak at $610 \mathrm{~nm}$ can be attributed to porphyrins, which has an excitation peak near $405 \mathrm{~nm}$ and an emission peak at $610 \mathrm{~nm}$ and is synthesised in liver (24). There was a weak $575 \mathrm{~nm}$ peak for both excitation wavelengths which could come from lipofuscin, a lipopigment present in the liver [25] which has a maximum fluorescence emission around $575 \mathrm{~nm}[26]$ and a concentration that increases with age.

At $405 \mathrm{~nm}$, spectral differences between disease states seem visually less apparent than at 375 $\mathrm{nm}$ excitation, although note that the difference is still statistically significant, as shown in the following sections. For this excitation wavelength, vitamin $A$ and $N A D(P) H$ are less strongly excited so their contributions to the fluorescence spectra will appear slightly less important than at $375 \mathrm{~nm}$ excitation. The spectral shape also results from the contributions of the other fluorophores present in the liver including flavins, [22], which have an emission band between $500 \mathrm{~nm}$ and 600nm [26], and arachidonic acid, which is a free fatty acid metabolized by the liver [23] that emits fluorescence between 400 and $550 \mathrm{~nm}$ [18].

\section{Data processing and analysis}

The results of the SMLR classification performed with a leave-one-out cross validation are presented in the confusion matrices in table 4 for the concatenated dataset. The overall classification accuracy obtained reached $95 \%$.

This table can be reinterpreted in terms of diagnostically more meaningful parameters by considering the sensitivity, specificity, positive and negative predictive values (PPV, NPV) of one group against the two others. The normal tissue (control group) could be differentiated from the mild steatotic and steatohepatitic tissue with $95.6 \%$ sensitivity, 96.8\% specificity, 94.8\% PPV and 96.8\% NPV. The steatotic tissue was discriminated from the normal and steatohepatitic tissue with $91.7 \%$ sensitivity, $96 \%$ specificity, $88 \%$ PPV, and 97.3\% NPV. Steatohepatitic tissue was separated from the two other groups with $96.5 \%$ sensitivity, 100\% specificity, 100\% PPV and $97.9 \%$ NPV.

The posterior probabilities calculated by SMLR of fluorescence spectra belonging to the correct disease group as classified by histopathology are plotted in figure 6 . Fifteen sites were spectrally misclassified with a probability below $50 \%$, with the remainder classified with an average probability of $98.9 \%$ indicating a strong confidence in the classification of the tissues. 


\section{Discussion}

This study aimed to assess fluorescence spectroscopy as a way to differentiate healthy livers from steatotic and steatohepatitic livers and compare its performance with ALT and TBARS assays.

Autofluorescence from liver has previously been studied in a tissue microscopy instrument under $366 \mathrm{~nm}$ laser excitation by Croce et al. In that study the analysis of the fluorescence spectra and photobleaching from rat livers enabled the determination of the fluorophores responsible for liver autofluorescence [33,34]. Among these, $\mathrm{NAD}(\mathrm{P}) \mathrm{H}$, vitamin $\mathrm{A}$ and collagen were identified as the primary markers to distinguish healthy, steatotic and fibrotic livers. Whilst this work was decisive for the understanding of liver fluorescence and opened perspectives on the potential of fluorescence spectroscopy as a tool to assess liver state, the instrumentation used in this work required the tissues to be excised, sectioned and prepared onto microscope slides. In another study, De Oliveira et al. acquired spectra in a rat liver model with different degrees of steatosis at $532 \mathrm{~nm}$ excitation using an optical fibre probe [34]. The most severe steatosis cases were clearly distinguished from the healthy ones by using the ratio of the fluorescence peak intensity at 605 $\mathrm{nm}$ over the backreflected excitation light intensity at $532 \mathrm{~nm}$. Moreover, this factor averaged within each study group is shown to follow linearly the fat concentration. However, consecutive study groups could not be differentiated one from another based on this ratio. The use of an algorithm utilising the whole spectrum, similar to SMLR, together with excitation at a wavelength that will allow autofluorescence from vitamin A, collagen, $\mathrm{NADH}$ and porphyrins is expected to improve the classification. This work noticeably showed the potential of fluorescence spectroscopy as a tool for NAFLD detection but did not directly assess its diagnosis performance.

The steatohepatitis group exhibited noticeably increased ALT and TBARS levels compared to the steatosis and control groups, leading to a clear differentiation of the steatohepatitic group with a p-value below 0.001 . However, no significant differences in these parameters were found between the control and steatotic groups and it was not possible to differentiate these two groups based on ALT and TBARS levels. Fluorescence emission spectra from different disease classes exhibited differences in their spectral shape as shown in figure 5 (A) and (B). The most visually prominent feature was the difference in fluorescence intensity at the peak (around $475 \mathrm{~nm}$ ) for $375 \mathrm{~nm}$ excitation. The normal, mild steatosis and steatohepatitis groups have steatosis scores of 0,2 and 3 respectively using NAS (see the "steatosis" column in table 1). The relative increase in intensity at $475 \mathrm{~nm}$ for $375 \mathrm{~nm}$ excitation from the normal (steatosis score 0 ) to the mild steatosis group (steatosis score 2, see figure $5 \mathrm{~A}$ ) is consistent with an increase in vitamin $\mathrm{A}$ content concomitant with the augmentation of lipids (20) accompanied by an increase in $N A D(P) H$, which has been shown to soar with steatosis (35).

The steatohepatitis group had a steatosis score of 3 and may therefore have been expected to have a higher intensity at $475 \mathrm{~nm}$ than the other groups. However, it actually has a lower intensity (Fig $5 \mathrm{~A}$ ), which may be due to the biochemical and biostructural changes induced by 
fibrosis in this model of steatohepatitis. Firstly, collagen which is induced by fibrosis is a well known scatterer and fluorophore which will affect the fluorescence and scattering properties of the liver tissue. Secondly, the contribution of vitamin A in fibrotic liver has been shown to be half that of healthy liver when excited at $366 \mathrm{~nm}$ [21]. Therefore, the presence of fibrotic tissue in the fibrosing steatohepatitic livers is likely to decrease the peak intensity in this group. Also, the evolution of $\mathrm{NAD}(\mathrm{P}) \mathrm{H}$ with fibrosing steatohepatitis is unknown. The difference between the spectra excited at $405 \mathrm{~nm}$ in the spectral interval from $450 \mathrm{~nm}$ to $500 \mathrm{~nm}$, was visually less obvious than for $375 \mathrm{~nm}$ excitation, although note that the difference is still significant, and that the results from the classification algorithm account for the response across the whole emission spectrum. Spectral differences between the $405 \mathrm{~nm}$ excitation spectra also stem from other liver fluorophores such as flavins and arachidonic acid.

The classification accuracy for single excitation was $80 \%$ and $95 \%$ for $375 \mathrm{~nm}$ and $405 \mathrm{~nm}$ excitation respectively. The classification with a combination of spectra at the two excitation wavelengths was found to be $95 \%$ and therefore did not improve the result obtained with the 405 $\mathrm{nm}$ laser diode only. This suggests that the optical system could be simplified by keeping only the $405 \mathrm{~nm}$ laser diode although the combination of the $375 \mathrm{~nm}$ and $405 \mathrm{~nm}$ might improve the classification performance with other models of NAFLD (data in preparation). Therefore, autofluorescence spectroscopy enabled the differentiation of the three groups with a high classification accuracy that could not be achieved with the ALT and TBARS levels. Moreover, the spectroscopic classification was nearly real time and hence significantly faster than the ALT and TBARS assays (typically 1 day) as well as histopathology (typically at least 2 days). Although in most cases fatty infiltration within the liver is relatively uniform, all diagnostic techniques used in the liver are open to sampling error. Fluorescence spectroscopy should be no more sampling error prone than biopsy, but has the added advantage that multiple readings may be taken without causing tissue damage and so any error may be minimised.

One advantage of the SMLR is that it computes the posterior probability of a spectrum belonging to a particular class, and as pointed out by Majumder [29], this can allow suspicious sites with less than $50 \%$ probability of being healthy to be identified and further examined. It could therefore be possible to diagnose NAFLD using fluorescence spectroscopy on freshly excised liver explants or biopsy material. Depending on the posterior probability of classification as healthy, the clinician could then decide on further histological examination. This procedure could help to speed up the diagnostic process as the result from spectroscopy is almost instantaneous, as well as having potential for intraoperative quantitative diagnosis and classification of NAFLD severity in donor liver tissue which may assist in graft selection in the transplant setting.

This result has been obtained in an animal model involving NAFLD mice only, where the contrast between disease states was unambiguous. In practice, NAFLD severity may need to be discriminated from other inflammatory conditions and from varying degrees of fibrosis or cirrhosis. The ability of fluorescence spectroscopy to distinguish NAFLD patients in these different situations remains to be tested. 


\section{Conclusion}

In summary, we have built a compact dual excitation spectroscopy system using laser diodes that can be used minimally invasively as the optical fibre probe has a $2 \mathrm{~mm}$ diameter. Fluorescence spectra at both excitation wavelengths were recorded from healthy, steatotic and steatohepatitic mice and analysed with SMLR. Excellent classification accuracy with strong confidence in the classification was obtained which support the use of fluorescence spectroscopy as a tool to quickly assess liver tissue graft ex vivo or in vivo at harvest.

\section{Acknowledgements}

Elements of this work have been funded by the EPSRC grants DT/F003064/1 and EP/E06342X/1 to DSE and an MRC Programme Grant to QMA, RDG \& MT. QMA is the recipient of a Clinical Senior Lectureship Award from the Higher Education Funding Council for England (HEFCE) and is a Newcastle NIHR BRC Investigator. RG and MRT are Imperial College NIHR BRC Investigators.

\section{References}

1. Anstee QM, Goldin RD. Mouse models in non-alcoholic fatty liver disease and steatohepatitis research. Int J Exp Pathol 2006; 87(1):1-16.

2. Saadeh S, Younossi ZM. The spectrum of nonalcoholic fatty liver disease: From steatosis to nonalcoholic steatohepatitis. Cleve Clin J Med 2000; 67(2):96-97.

3. El-Zayadi AR. Hepatic steatosis: A benign disease or a silent killer. World Journal of Gastroenterology 2008; 14(26):4120-4126.

4. Schreuder T, Verwer BJ, van Nieuwkerk CMJ, Mulder CJJ. Nonalcoholic fatty liver disease: An overview of current insights in pathogenesis, diagnosis and treatment. World Journal of Gastroenterology 2008; 14(16):2474-2486.

5. Browning JD, Szczepaniak LS, Dobbins R, Nuremberg P, Horton JD, Cohen JC, Grundy $\mathrm{SM}$, Hobbs HH. Prevalence of hepatic steatosis in an urban population in the United States: Impact of ethnicity. Hepatology 2004; 40(6):1387-1395.

6. Lian Tock ARD, Aline de Piano, et al. Long-Term Effects of Metformin and Lifestyle Modification on Nonalcoholic Fatty Liver Disease Obese Adolescents. Journal of Obesity 2010; vol. 2010.

7. WHO. Obesity and overweight. 2006; Available from: http://www.who.int/mediacentre/factsheets/fs311/en/index.html.

8. WHO. Diabetes - Key facts. 2010

9. Hashimoto $\mathrm{K}$, Miller $\mathrm{C}$. The use of marginal grafts in liver transplantation. J Hepatobiliary Pancreat Surg 2008; 15(2):92-101.

10. Kim SH, Lee JM, Han JK, Lee JY, Lee KH, Han CJ, Jo JY, Yi NJ, Suh KS, Shin KS, Jo SY, Choi BI. Hepatic macrosteatosis: Predicting appropriateness of liver donation by using MR imaging - Correlation with histopathologic findings. Radiology 2006; 240(1):116-129.

11. Nocito A, El-Badry AM, Clavien PA. When is steatosis too much for transplantation? J Hepatol 2006; 45(4):494-499.

12. Bravo AA, Sheth SG, Chopra S. Current concepts: Liver biopsy. N Engl J Med 2001; 344(7):495-500.

13. Graif M, Yanuka M, Baraz M, Blank A, Moshkovitz M, Kessler A, Gilat T, Weiss J, Walach $\mathrm{E}$, Amazeen $\mathrm{P}$, Irving CS. Quantitative estimation of attenuation in ultrasound video images - Correlation with histology in diffuse liver disease. Invest Radiol 2000; 35(5):319324. 
14. Cheruku SM, Fishbein MH. Hepatic MRI for fat quantitation: Its relationship to fat morphology, diagnosis and ultrasound. Gastroenterology 2004; 126(4):A758-A758.

15. Foucher J, Castera L, Bernard PH, Adhoute X, Laharie D, Bertet J, Couzigou P, de Ledinghen $V$. Prevalence and factors associated with failure of liver stiffness measurement using FibroScan in a prospective study of 2114 examinations. Eur $\mathrm{J}$ Gastroenterol Hepatol 2006; 18(4):411-412.

16. Clark JM, Diehl AM. Nonalcoholic fatty liver disease - An underrecognized cause of cryptogenic cirrhosis. Jama-Journal of the American Medical Association 2003; 289(22):3000-3004.

17. Ramanujam N. Fluorescence spectroscopy of neoplastic and non-neoplastic tissues. Neoplasia 2000; 2(1-2):89-117.

18. Croce AC, Ferrigno A, Vairetti M, Bertone R, Freitas I, Bottiroli G. Autofluorescence properties of isolated rat hepatocytes under different metabolic conditions. Photochemical \& Photobiological Sciences 2004; 3(10):920-926.

19. Croce AC, De Simone U, Vairetti M, Ferrigno A, Bottiroli G. Autofluorescence properties of rat liver under hypermetabolic conditions. Photochemical \& Photobiological Sciences 2007; 6:1202-1209.

20. Croce AC, De Simone U, Vairetti M, Ferrigno A, Boncompagni E, Freitas I, Bottiroli G. Liver autofluorescence properties in animal model under altered nutritional conditions. Photochemical \& Photobiological Sciences 2008; 7(9):1046-1053.

21. Croce AC, De Simone U, Freitas I, Boncompagni E, Neri D, Cillo U, Bottiroli G. Human Liver Autofluorescence: An Intrinsic Tissue Parameter Discriminating Normal and Diseased Conditions. Lasers Surg Med 2010; 42(5):371-378.

22. Yates CA, Evans GS, Powers HJ. Riboflavin deficiency: early effects on post-weaning development of the duodenum in rats. Br J Nutr 2001; 86(5):593-599.

23. Capdevila J, Chacos N, Werringloer J, Prough RA, Estabrook RW. Liver Microsomal Cytochrome-P-450 and the Oxidative-Metabolism of Arachidonic-Acid. Proceedings of the National Academy of Sciences of the United States of America-Biological Sciences 1981; 78(9):5362-5366.

24. Bloomer JR. Liver metabolism of porphyrins and haem. J Gastroenterol Hepatol 1998; 13(3):324-329.

25. Porta EA. Ceroid and Lipofuscin in the Liver - History and Its State-of-the-Art. Liver and Aging - 1990 1991; 940:89-105

26. Wagnieres GA, Star WM, Wilson BC. In vivo fluorescence spectroscopy and imaging for oncological applications. Photochem Photobiol 1998; 68(5):603-632.

27. Krishnapuram B, Carin L, Figueiredo MAT, Hartemink AJ. Sparse multinomial logistic regression: Fast algorithms and generalization bounds. leee Transactions on Pattern Analysis and Machine Intelligence 2005; 27(6):957-968.

28. Bosko J, Edwards M, Artemink A. SMLR: Sparse Multinomial Logistic Regression. Available from: http://www.cs.duke.edu/ amink/software/smlr/.

29. Majumder SK, Gebhart S, Johnson MD, Thompson R, Lin WC, Mahadevan-Jansen A. A probability-based spectroscopic diagnostic algorithm for simultaneous discrimination of brain tumor and tumor margins from normal brain tissue. Applied Spectroscopy 2007; 61(5):548-557.

30. Keller MD, Majumder SK, Kelley MC, Meszoely IM, Boulos FI, Olivares GM, MahadevanJansen A. Autofluorescence and Diffuse Reflectance Spectroscopy and Spectral Imaging for Breast Surgical Margin Analysis. Lasers Surg Med 2010; 42(1):15-23.

31. Tibshirani R. Regression shrinkage and selection via the Lasso. Journal of the Royal Statistical Society Series B-Methodological 1996; 58(1):267-288.

32. Kleiner DE, Brunt EM, Van Natta M, Behling C, Contos MJ, Cummings OW, Ferrell LD, Liu YC, Torbenson MS, Unalp-Arida A, Yeh M, McCullough AJ, Sanyal AJ, Clin NS. Design and validation of a histological scoring system for nonalcoholic fatty liver disease. Hepatology 2005; 41(6):1313-1321.

33. Sankarankutty AK, Silva OCE, Ferreira J, Souza MEJ, Gomes MC, Kurachi C, Bagnato VS. Use of laser auto-fluorescence for evaluating liver grafts. Laser Physics Letters 2006; 3(11):539-545.

34. De Oliveira GR, Sankarankutty AK, Castro e Silva O, Ferreira J, Kurachi C, Zucoloto S, Vannucchi H, Jordao AA, Marchini JS, Bagnato VS. Fluorescence spectroscopy to diagnose hepatic steatosis in a rat model of fatty liver. Liver International 2009; 29(3):331-336. 
35. Sun $\mathrm{CK}$, Zhang $\mathrm{XY}$, Wheatley $\mathrm{AM}$. Increased $\mathrm{NAD}(\mathrm{P}) \mathrm{H}$ fluorescence with decreased blood flow in the steatotic liver of the obese Zucker rat. Microvasc Res 2003; 66(1):15-21. 


\section{Legends}

Figure 1: Experimental set-up of multi excitation wavelength fluorescence spectroscopy probe.

Figure 2: Typical H\&E stained liver sections at $x 400$ magnification showing (A) Db/m mouse liver fed standard Chow., (B) Db/Db mouse liver fed standard Chow showing steatosis only, (C) Db/Db mouse liver fed MCD showing steatosis, lobular inflammation and hepatocyte ballooning.

Figure 3: Mean malondialdehyde concentration in the livers of the mice \pm SEM.

Figure 4: Mean ALT levels in the plasma of the mice \pm SEM.

Figure 5: Mean spectra at (A) $375 \mathrm{~nm}$ and (B) $405 \mathrm{~nm}$ excitation and error bars representing standard deviation.

Figure 6: Posterior probability of fluorescence spectra from interrogated tissue sites belonging to their correct disease group (as classified by histopathology): control (114 sites), mild steatosis (72 sites), steatohepatitis (114 sites).

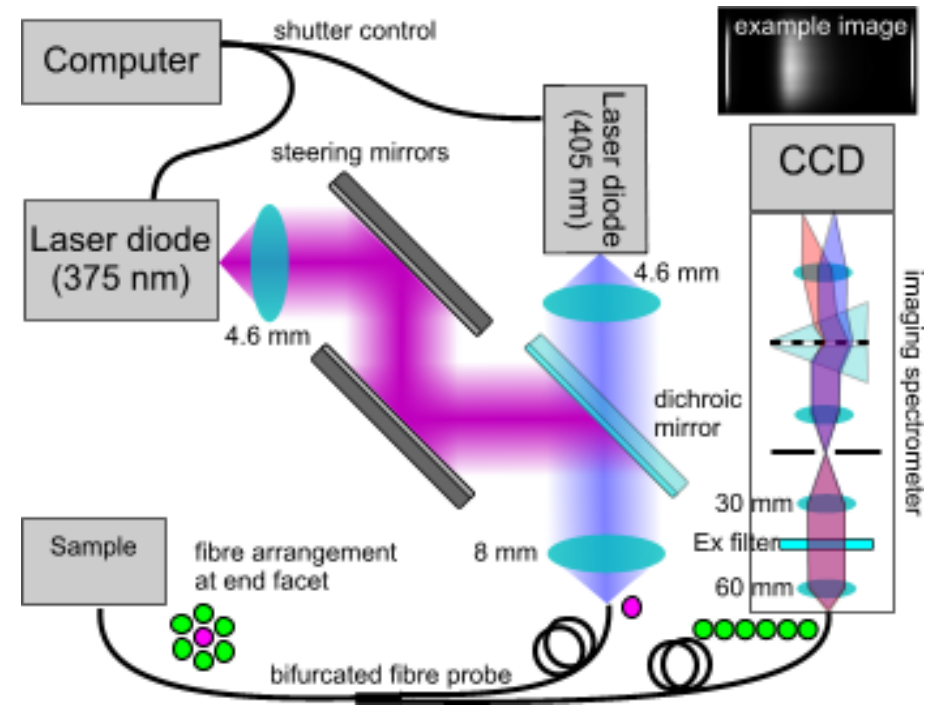

Figure 2: Experimental set-up of multi excitation wavelength fluorescence spectroscopy probe. 

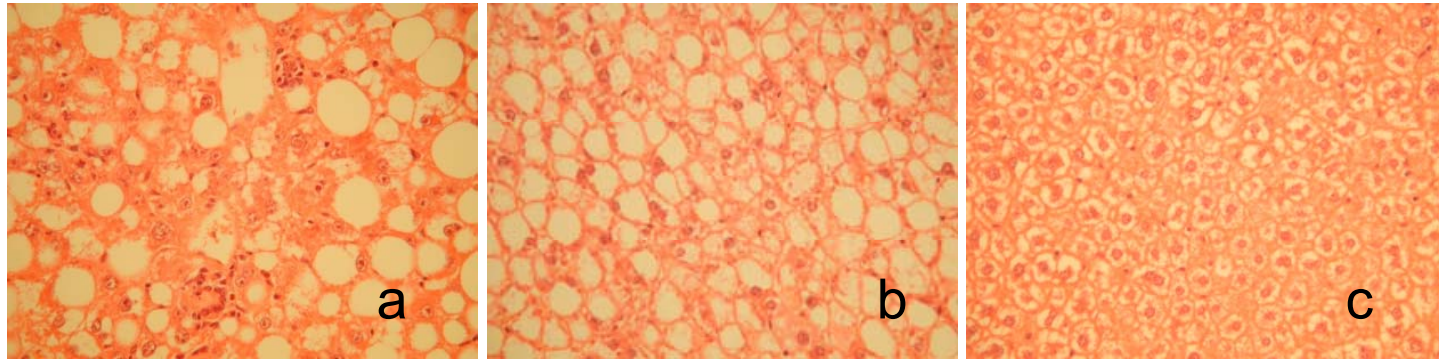

Figure 3 typical H\&E stained liver sections at x 400 magnification showing a) Db/Db mouse liver fed MCD showing steatosis, lobular inflammation and hepatocyte ballooning, b) Db/Db mouse liver fed standard Chow showing steatosis only, c) Db/m mouse liver fed standard Chow.

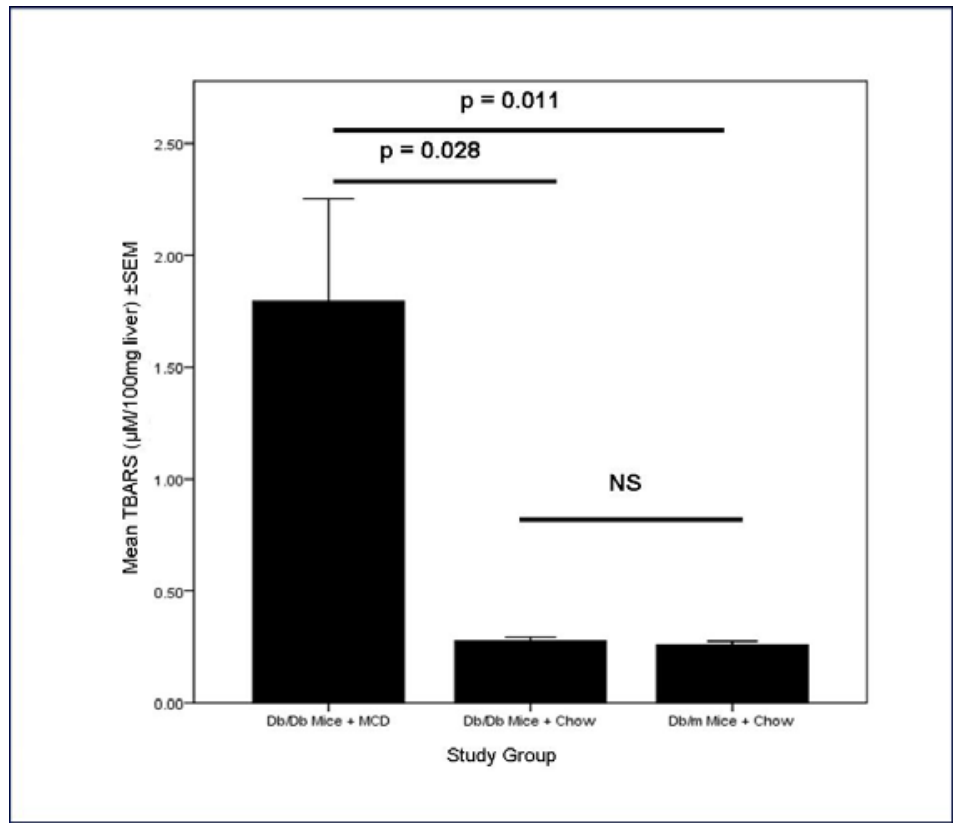

Figure 4: Mean malondialdehyde concentration in the livers of the mice \pm SEM 


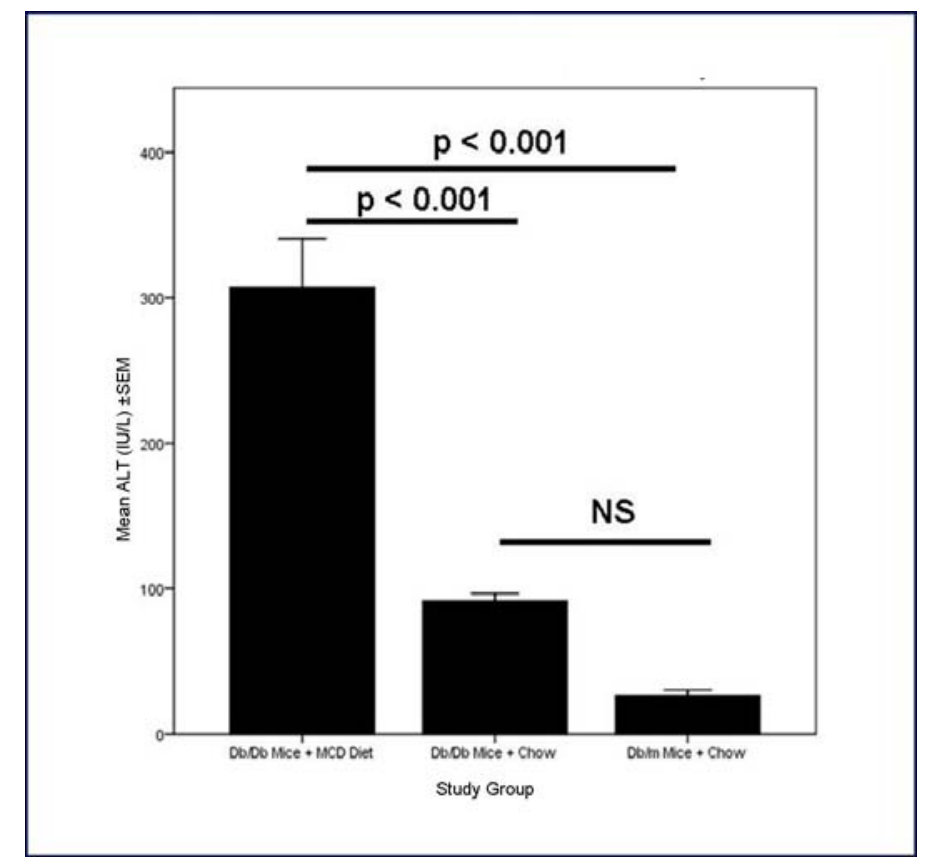

Figure 5. Mean ALT levels in the plasma of the mice \pm SEM
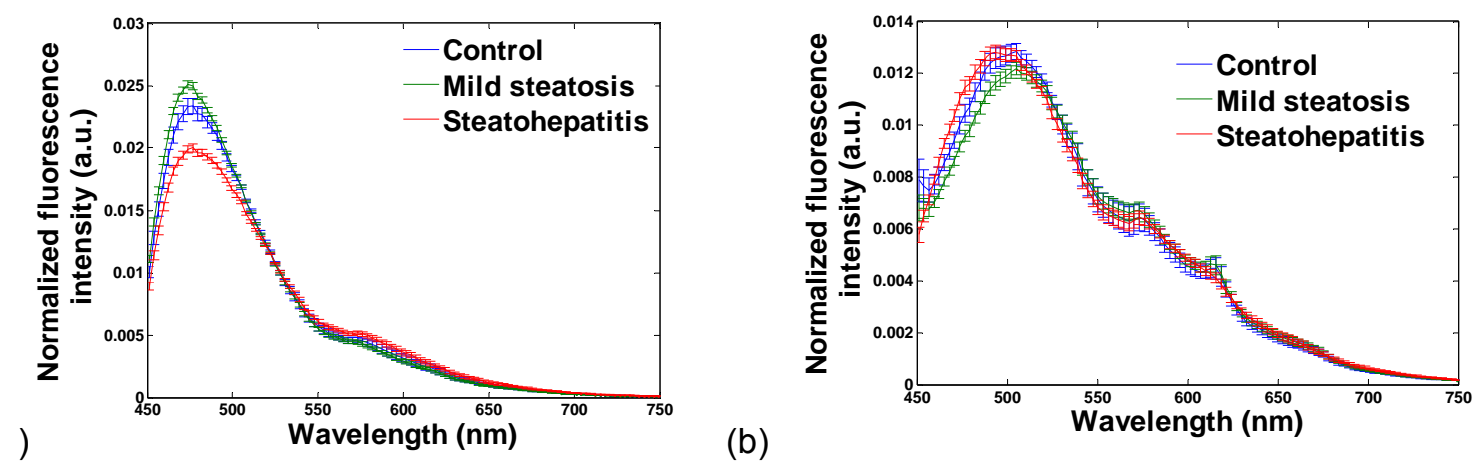

Figure 6: Mean spectra at (a) $375 \mathrm{~nm}$ and (b) $405 \mathrm{~nm}$ excitation and error bars representing standard deviation. 


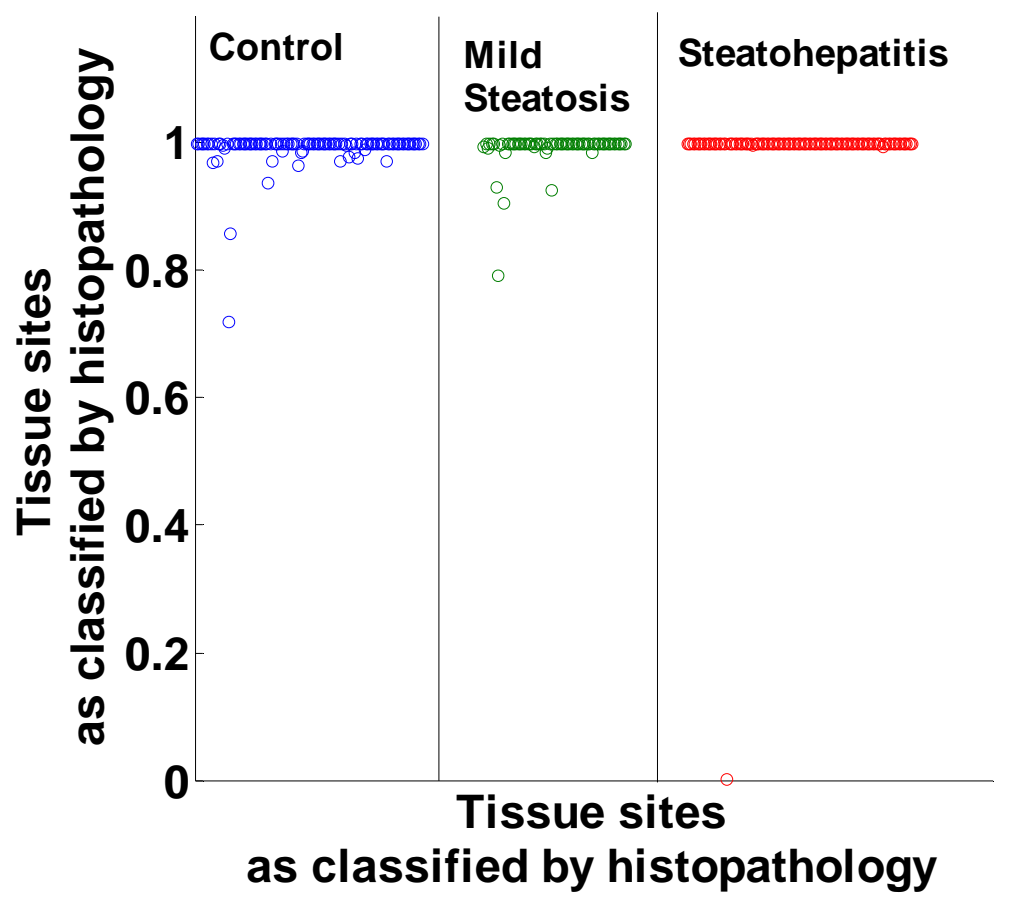

Figure 7: Posterior probability of fluorescence spectra from interrogated tissue sites belonging to their correct disease group (as classified by histopathology): steatohepatitis (114 sites), mild steatosis (72 sites), control (114 sites).

\begin{tabular}{lc}
\hline Disease group & $\begin{array}{c}\text { Number of } \\
\text { measurements }\end{array}$ \\
\hline Steatohepatitis (Group A) & 114 \\
Mild steatosis (Group B) & 72 \\
Control (Group C) & 114
\end{tabular}

Table 1: Number of tissue fluorescence measurements per disease group.

\begin{tabular}{lcccc}
\hline Study Group & Steatosis & $\begin{array}{c}\text { Lobular } \\
\text { inflammation }\end{array}$ & $\begin{array}{c}\text { Hepatocyte } \\
\text { ballooning }\end{array}$ & $\begin{array}{c}\text { NAFLD activity } \\
\text { score }\end{array}$ \\
\hline Steatohepatitis group A & 3 & 2 & 2 & 7 \\
\hline Mild steatosis group B & 2 & 0 & 0 & 2 \\
\hline Control group C & 0 & 0 & 0 & 0 \\
\hline
\end{tabular}

Table 2. Median NAFLD activity score for the mice. 


\begin{tabular}{ll}
\hline Pilot Study Group & $\begin{array}{l}\text { Malondialdehyde } \\
(\mu \mathrm{M} \text { per 100mg of liver }) \pm \mathrm{SEM}\end{array}$ \\
\hline $\begin{array}{l}\mathrm{Db} / \mathrm{Db} \text { mice on MCD diet } \\
\text { Steatohepatitis group A }\end{array}$ & $1.79 \pm 0.46$ \\
\hline Db/Db mice on standard chow & $0.28 \pm 0.02$ \\
Mild steatosis group B & \\
\hline $\begin{array}{l}\text { Db/m mice on standard chow } \\
\text { Control group C }\end{array}$ & $0.26 \pm 0.02$ \\
\hline
\end{tabular}

Table 3: Mean malondialdehyde concentration in the livers of the mice \pm SEM

\begin{tabular}{ll}
\hline Pilot Study Group & ALT biochemistry (IU/L) \pm SEM \\
\hline $\begin{array}{l}\text { Db/Db mice on MCD diet } \\
\text { Steatohepatitis group A }\end{array}$ & $307.34 \pm 33.38$ \\
\hline Db/Db mice on standard chow & $91.77 \pm 4.78$ \\
Mild steatosis group B & \\
\hline Db/m mice on standard chow & $26.40 \pm 4.07$ \\
Control group C & \\
\hline
\end{tabular}

Table 4. Mean ALT levels in the plasma of the mice \pm SEM.

\begin{tabular}{lcccc}
\hline & & \multicolumn{3}{c}{ Fluorescence spectroscopy } \\
\cline { 3 - 5 } & Disease group & Steatohepatitis & Mild steatosis & Control \\
\hline \multirow{3}{*}{ Histopathology } & Steatohepatitis & 113 & 1 & 0 \\
& Mild steatosis & 0 & 72 & 0 \\
& Control & 0 & 0 & 114 \\
\hline
\end{tabular}

Table 5:Confusion matrix presenting the ability of fluorescence spectroscopy to distinguish between control, moderate steatosis and steatohepatitis disease groups. 